\title{
Patient-reported quality of life and pain after permissive weight bearing in surgically treated trauma patients with tibial plateau fractures: a retrospective cohort study
}

\author{
Pishtiwan Hassan Shaker Kalmet ${ }^{1} \cdot$ Yvette Y. Van Horn $^{2}$ - Sebastian Sanduleanu ${ }^{1}$. Henk A. M. Seelen ${ }^{3,4}$. \\ Peter R. G. Brink ${ }^{1} \cdot$ Martijn Poeze ${ }^{1,5}$
}

Received: 22 May 2018 / Published online: 6 December 2018

(c) The Author(s) 2018

\begin{abstract}
Introduction A Dutch survey among orthopedic surgeons and trauma surgeons showed that almost $90 \%$ of the surgeons do not follow protocols regarding the weight bearing aftercare for tibial plateau fractures. Clinical studies comparing permissive weight bearing (PWB) versus restricted weight bearing (RWB) after surgically treated tibial plateau fractures are not available. The aim of this study was to inventory potential differences in quality of life and pain, and number of complications in patients with surgically treated tibial plateau fractures who followed a PWB regime, relative to those that followed a RWB regime.

Materials and methods This retrospective cohort study included surgically treated trauma patients with tibial plateau fractures, who underwent rehabilitation according to PWB or RWB between 2005 and 2015. Data such as demographics, patientreported quality of life and pain, and patient outcome were collected.

Results This cohort study included 91 patients with a tibial plateau fracture (31 and 60 patients in the PWB and RWB groups respectively). No significant between-group differences in either age or gender were found. However, a significant difference in fracture type was found between groups, $(p=0.04)$. No significant differences were found in either patient-reported SF-12 or VAS scores between the PWB group and RWB group. Time to full weight bearing was significantly shorter in the PWB than in the RWB group, i.e., 14.7 versus 20.7 weeks, $(p=0.02)$. No significant differences were found regarding postoperative complications between the PWB and the RWB groups, i.e., $6.5 \%$ versus $10.0 \%$, respectively.

Conclusion PWB after surgically treated tibial plateau fractures is safe and is related to a significantly reduced time to full weight bearing with no significant differences in patient-reported quality of life and pain or complication rates.
\end{abstract}

Keywords Tibial plateau fractures $\cdot$ Trauma patients $\cdot$ Complications $\cdot$ Rehabilitation $\cdot$ Weight bearing $\cdot$ Quality of life

Pishtiwan Hassan Shaker Kalmet

pishtiwan.kalmet@mumc.nl

1 Department of Traumatology, Trauma Surgery, Maastricht University Medical Center, P. Debyelaan 25, 6229, HX, Maastricht, The Netherlands

2 Department of Amputation, Traumatology and Orthopaedics, Adelante Rehabilitation Center, Hoensbroek, The Netherlands

3 Adelante Center of Expertise in Rehabilitation and Audiology, Hoensbroek, The Netherlands

4 Research School CAPHRI, Department of Rehabilitation Medicine, Maastricht University, Maastricht, The Netherlands

5 Nutrim School for Nutrition, Toxicology and Metabolism, Maastricht University, Maastricht, The Netherlands

\section{Introduction}

The incidence of patients with tibial plateau fractures is approximately 13.3 per 100,000 [1]. Protocols for postoperative management of tibial plateau fractures were formulated about 60 years ago and suggest non- or partial weight bearing [2]. A survey about the adherence of current protocols showed that almost $90 \%$ of the surgeons do not follow these protocols standardly regarding the weight bearing aftercare for tibial plateau fractures [3]. In addition, patient's compliance to a non- or partial weight bearing regimen is found to be poor and highly depending on the age of the patient [4, 5]. Elderly patients seem to be unable to maintain weightbearing restrictions [6]. Thus, patients are likely to start 
weight bearing in an earlier phase than prescribed in current protocols.

The postoperative management of these surgically treated tibial plateau fractures in trauma patients is also very important regarding the functional outcome. The average overall postoperative complication rate in tibial plateau fractures, combining implant failures, secondary dislocation, nonunion and infections into a composite end-point, is around 4-27\% according to literature [7-14].

The standard aftercare treatment in surgically treated trauma patients with fractures of the tibial plateau features is non- or partial-weight bearing [15]. According to the Arbeitsgemeinschaft für Osteosynthesefragen $(\mathrm{AO})$ principles of fracture management, postoperative management of tibial plateau fractures generally consists of toe-touch weight bearing for 6-8 weeks. As to fractures caused by extremely highenergy impact, these patients may need to adhere to toetouch weight bearing regimen for 10-12 weeks [2]. There is currently no consensus among surgeons worldwide with regard to early weight bearing (i.e., permissive weight bearing) versus restricted weight bearing in surgically treated trauma patients with fractures of the tibial plateau [16].

Biomechanical and animal studies indicate that early weight bearing is beneficial [17-19], but high-quality clinical studies comparing permissive weight bearing (PWB) versus restricted weight bearing (RWB) after surgically treated tibial plateau fractures are scarce.

The aim of the present study was to inventory potential differences in quality of life and pain, and number of complications in patients with surgically treated tibial plateau fractures who followed a permissive weight bearing regime, relative to those that followed a restricted weight bearing regime.

\section{Patients and methods}

This retrospective cohort study included surgically treated trauma patients with tibial plateau fractures at Maastricht University Medical Center+, the Netherlands, who underwent aftercare according the PWB or a RWB protocol between 2005 and 2015. In the PWB group, the patients were discharged to a rehabilitation center, where they were treated according the PWB protocol. Since 2003 PWB was gradually implemented and became standard care in our rehabilitation center from 2005. The fracture aftercare process starts by assessing the patient's profile. Next, the generic and patient-specific treatment goals are identified, which, when combined, lead to the aftercare treatment aims. These aftercare treatment aims are then contrasted to the patient's profile descriptors, which, together with potential predictors of surgically treated fracture aftercare outcome, may give insight into (a) the feasibility of the aftercare treatment aims; (b) the estimated time frame in which the aftercare treatment aims may be reached; and (c) the intensity/dosage/weight bearing needed to achieve the aftercare treatment aims. The increase in weight bearing is not based on a fixed percentage per week: weight bearing is gradually increased, based on the patient's clinical presentation and with special attention to the quality of gait. Other key elements include body awareness and safe patient handling and moving algorithms, which are also considered to be key factors for successful treatment. The program involves multidisciplinary cooperation with surgeons, rehabilitation physicians and physical therapists, which is considered paramount to safely use the PWB protocol.

The patients included in the protocol suffered from two or more fractures (upper and lower extremity fractures), and therefore, needed more aftercare. The patients in the RWB group were discharged to their own home. They received passive exercise to maintain the muscles and the knee joint supported by a physical therapist, as prescribed by the surgeon.

All data in the study were collected from the electronic medical records by one researcher. Demographics of patients included age, gender and the presence of other fractures at the same time.

Primary outcome measures included the patient-reported questionnaire after at least 1-year follow-up; (1) Quality of life measured with the Short Form 12 (SF-12) [20]. The SF-12 consists of 12 items that assess eight dimensions of health: physical functioning, role-physical, bodily pain, general health, vitality, social functioning, role-emotional and mental health. The SF-12 measures various aspects of physical and mental health from which physical and mental summary scores can be calculated. (2) The intensity of pain measured with the VAS scale, ( 0 is no pain and 10 is worst pain) [21].

Time from surgery till full weight bearing and the total number and type of postoperative complications were collected from the electronic medical records. A postoperative complication was defined as a composite end-point comprising any complication, related to the fracture, that occurred during the aftercare regimen, these were recorded as either present or not present, along with the type of complications.

The medical ethics committee of Zuyderland Medical Center, Heerlen, the Netherlands approved this study and informed consent was given by all patients.

\section{Statistical analysis}

Statistical analysis was performed with IBM SPSS Statistics, Version 23.0, Armonk, New York. Descriptive statistics were used to describe the demographic data and baseline characteristics of the entire population. Independent samples 
$t$ tests were used for normally distributed continuous data and Chi-squared tests for categorical variables. Results are presented as either mean \pm standard deviation $(\mathrm{SD})$ or as frequencies and percentages. In case of non-parametric data the median with the interquartile range (IQR) are described. Binary logistic regression was performed to assess independent predictors of late full weight bearing ( $>12$ weeks) throughout both PWB and RWB groups. The level of statistical significance was set at $\alpha=0.05$.

\section{Results}

\section{Baseline characteristics}

This cohort study included 91 patients, 31 of whom were in the PWB group and 60 in the RWB group. Characteristics of patients in the PWB group and RWB group are presented in Table 1. Patients in the PWB group were significantly more likely to have a more complex fracture type [Schatzker fracture type (IV-VI) [22]] $(p=0.04)$ and more concomitant fractures than those in the RWB group $(p<0.01)$. No differences in age or gender were found between the two groups. Furthermore, no differences were found in surgical procedures between the two groups.

\section{Patient-reported quality of life and pain}

The overall response rate of the patient-reported questionnaire SF-12 and VAS scale was 72.5\% (i.e., 66/91). No significant difference was found in response rate between the PWB group (80.6\%) and RWB $(68.3 \%)$ group $(p=0.32)$. The time between surgery and the moment at which the questionnaires administered was significantly higher in the RWB group than in the PWB group: 7.6 (3.2) years versus $4.6(2.4)$ years $(p<0.01)$. No significant between-group differences were found in either quality of life measured with the SF-12 or the pain measured with the VAS scale (Table 2).

From the total population $38.5 \%$ of the patients $(N=35)$ reached full weight bearing within 12 weeks. The number of patients who reached full weight bearing within 12 weeks
Table 2 Functional outcome measurements in the PWB and RWB groups

\begin{tabular}{|c|c|c|c|c|}
\hline & $\mathrm{PWB}(N=25)$ & RWB $(N=41)$ & Total $(N=66)$ & $p$ \\
\hline $\begin{array}{c}\text { Mean SF-12 } \\
\text { (quality of } \\
\text { life) (SD) }\end{array}$ & $58.0(20.7)$ & $68.8(23.1)$ & 64.7 (22.7) & 0.06 \\
\hline $\begin{array}{l}\text { Mean VAS } \\
\text { scale } \\
\text { (pain) } \\
\text { (SD) }\end{array}$ & $3.6(2.2)$ & $2.8(2.7)$ & $3.1(2.5)$ & 0.24 \\
\hline
\end{tabular}

$S D$ standard deviation

was significantly higher in the PWB group than in the RWB group: $58.1 \%$ versus $28.3 \%(p<0.01)$. Time from surgery to ascertainment of full weight bearing was significantly shorter in the PWB group than in the RWB group: 14.7 (11.6) weeks versus 20.7 (11.5) weeks ( $p=0.02$ ) (Table 3). Binary logistic regression analysis revealed that, irrespective of PWB or RWB, Schatzker type and multiple fractures $(p<0.05)$ were independent predictors of late full weight bearing ( $>12$ weeks). No significant differences were found in time from surgery to full weight bearing between the specific fracture types (Schatzker type I-III versus Schatzker type IV-VI) $(p=0.10)$ in the PWB group (Table 4).

\section{Patient outcome}

No significant differences were found in the incidence of postoperative complications between the PWB group and the RWB group, values of which were $6.5 \%(N=2)$ versus $10.0 \%(N=6)$, respectively. In the PWB group. The complications in the PWB group consisted of $N=1$ non-union and $N=1$ superficial wound infection. It should be noted, however, that both patients started full weight bearing after the postoperative complication. The complications in the RWB group consisted of $N=3$ non-unions, $N=2$ superficial wound infections and $N=1$ deep infection. Furthermore, no significant differences between the PWB group and RWB group were found regarding either the postoperative removal of osteosynthesis material or the number of total knee prostheses (Table 5).
Table 1 Baseline characteristics of the PWB and RWB groups

\begin{tabular}{lccrr}
\hline & PWB $(N=31)$ & RWB $(N=60)$ & Total $(N=91)$ & $p$ \\
\hline Female & $12(38.7 \%)$ & $27(45.0 \%)$ & $39(42.9 \%)$ & 0.66 \\
Mean age (SD), years & $50.4(12.6)$ & $50.9(12.4)$ & $50.8(12.4)$ & 0.86 \\
$\geq 2$ fractures & $26(83.9 \%)$ & $5(8.3 \%)$ & $31(34.1 \%)$ & $<0.01$ \\
Schatzker types & & & & \\
$\quad$ Type I-III & $7(22.6 \%)$ & $27(45.0 \%)$ & $34(37.4 \%)$ & 0.04 \\
Type IV-VI & $24(77.4 \%)$ & $33(55.0 \%)$ & $57(62.6 \%)$ & \\
\hline
\end{tabular}

$S D$ standard deviation 
Table 3 Time to full weight bearing in the PWB and RWB groups

\begin{tabular}{lccrr}
\hline & PWB $(N=31)$ & RWB $(N=60)$ & Total $(N=91)$ & \multicolumn{1}{c}{$p$} \\
\hline Within 12 weeks & $18(58.1 \%)$ & $17(28.3 \%)$ & $35(38.5 \%)$ & $<0.01$ \\
$\begin{array}{l}\text { Mean time to full weight bear- } \\
\text { ing (SD), in weeks }\end{array}$ & $14.7(11.6)$ & $20.7(11.5)$ & $18.6(11.9)$ & 0.02 \\
\hline
\end{tabular}

$S D$ standard deviation

\begin{tabular}{lcccc}
\hline & $\begin{array}{l}\text { Schatzker } \\
\text { Type I-III }(N=7)\end{array}$ & $\begin{array}{l}\text { Schatzker } \\
\text { Type IV-VI }(N=24)\end{array}$ & $\begin{array}{l}\text { Total } \\
\text { Type I-VI }(N=31)\end{array}$ & $p$ \\
\hline Within 12 weeks & $6(85.7 \%)$ & $12(50.0 \%)$ & $18(58.1 \%)$ & 0.10 \\
$\begin{array}{l}\text { Mean time to full weight } \\
\text { bearing (SD), in weeks }\end{array}$ & $8.3(5.1)$ & $16.5(12.4)$ & $14.7(11.6)$ & 0.10 \\
\hline
\end{tabular}

$S D$ standard deviation
Table 4 Time to full weight bearing for specific fracture types in the PWB group follow the restrictions in weight bearing and advance their weight bearing as fracture healing progresses.

During normal daily activities the knee joint experiences forces between 220 and $350 \%$ of a person's body weight. As even a $3-\mathrm{mm}$ step-off in the tibial plateau can increase the cartilage contact stresses by $75 \%$, concerns are raised that loss of reduction could lead to worse patient outcomes, even in case of non-weight bearing [24]. On the other hand, it is often stated that early weight bearing does not pose an undue risk of complications or worse patient outcomes compared to a non-weight bearing protocol, as reported in a recent randomized controlled trial dealing with fractures of the ankle joint [25]. These two statements are contradictory and require further elaboration. Our study adds evidence in favor of regimens with earlier than standard postoperative weight bearing protocols and shows that there is no significant difference in quality of life, pain or complications compared to RWB.

One of the key objections against early weight bearing is the possibility of fracture displacement [26]. In one radiostereometric study at 1 year after early weight bearing of fractures of the tibia plateau, the mean craniocaudal migration of the fracture fragments was $-0.34 \mathrm{~mm}(-1.64$ to 1.51$)$ [27]. This case series has shown that, in the Schatzker type II fractures investigated, internal fixation with subchondral screws and a buttress plate provided adequate stability to allow immediate postoperative partial weight-bearing, without harmful consequences.

Longer term outcomes have as well been described in the literature, with more favorable results for PWB. In a prospective, multicenter randomized trial involving bicondylar tibial plateau fractures, a group of 43 patients underwent fixation with external ring fixation and were permitted to bear full weight, while a group of 40 patients underwent open reduction and internal fixation with restricted weight bearing [8]. At a minimum 2-year follow-up, there was no 
difference in reoperations, articular incongruity, or development of radiographic signs of osteoarthritis between the two groups. In line with this study, our study found that there were no significant differences in pain or reoperations (removal of osteosynthesis material or implants of total knee prostheses). Interestingly, removal of osteosynthesis material in the PWB group was lower than in RWB group, i.e., 22.6\% versus $40.0 \%$, respectively.

According to recent literature, a composite postoperative complication rate of up to $27 \%$ has been reported in tibial plateau fractures [7-14]. Comparing our complication data with data published in recent literature, we found decreased rates of postoperative complication in tibial plateau fractures treated by means of a PWB protocol, despite the fact that more severe fractures were found in our PWB population. The latter could be an explanation for the fact that the other $41.9 \%$ of the PWB population did not reach full weight bearing within 12 weeks, which might be due to a highcomorbidity rate of our PWB population. Nevertheless, the average time to full weight bearing was significantly lower in the PWB group than the RWB group.

Over and under-loading may lead to prolonged and complicated recovery. A certain minimum level of loading is required to elicit micro-movements between adjacent bony fracture components, stimulating biological processes that enhance fracture consolidation and minimizing effects of immobilization $[28,29]$. To optimize recovery with the lowest number of complications we want to set out a treatment that is near to the upper boundary of the therapeutic bandwidth regarding weight bearing, yet safe enough to avoid complications regarding overloading.

Our study, the first study comparing PWB with RWB, adds evidence in support of the use of PWB in patients with surgically treated tibial plateau fractures. However, limitations in our study include the retrospective nature of the study and, due to this retrospection, not taking into account surgeon-oriented functional outcome scores (e.g., knee function) or generic patient satisfaction scores. Furthermore, no radiological controls have been done to investigate the alignment of the fractures. Another limitation of the study is the lack of monitoring patient compliance. To mitigate these limitations, we have started a prospective cohort study in patients with fractures of the lower extremities [30].

\section{Conclusion}

This retrospective cohort study shows that permissive weight bearing after surgically treated tibial plateau fractures is safe and is related to a significant reduced time to full weight bearing with no significant differences in patient-reported quality of life and pain or complication rates.
Funding There is no funding source.

\section{Compliance with ethical standards}

Conflict of interest The authors declare that they have no conflict of interest.

Ethical approval This article does not contain any studies with human participants or animals performed by any of the authors. The medical ethics committee of Zuyderland Medical Center, Heerlen, the Netherlands approved this study and informed consent was given by all patients.

Open Access This article is distributed under the terms of the Creative Commons Attribution 4.0 International License (http://creativeco mmons.org/licenses/by/4.0/), which permits unrestricted use, distribution, and reproduction in any medium, provided you give appropriate credit to the original author(s) and the source, provide a link to the Creative Commons license, and indicate if changes were made.

\section{References}

1. Court-Brown CM, Caesar B (2006) Epidemiology of adult fractures: a review. Injury 37(8):691-697

2. Ru“edi TP, Buckley RE, Moran CG (2007) AO principles of fracture management, 2nd edn. Thieme, New York, p 832

3. van der Vusse M, Kalmet PHS, Bastiaenen CHG et al (2017) Is the AO guideline for postoperative treatment of tibial plateau fractures still decisive? A survey among orthopaedic surgeons and trauma surgeons in the Netherlands. Arch Orthop Trauma Surg 137(8):1071-1075

4. Hurkmans HL, Bussmann BJ, Selles RW et al (2007) The difference between actual and prescribed weight bearing of total hip patients with a trochanteric osteotomy: long-term vertical force measurements inside and outside the hospital. Arch Phys Med Rehabil 88:200-206

5. Hustedt JW, Blizzard DJ, Baumgaertner MR et al (2012) Is it possible to train patients to limit weight bearing on a lower extremity? Orthopedics 35:e31-e37

6. Kammerlander C, Pfeufer D, Lisitano LA et al (2018) Inability of older adult patients with hip fracture to maintain postoperative weight-bearing restrictions. J Bone Jt Surg Am 100:936-941

7. Basques BA, Webb ML, Bohl DD et al (2015) Adverse events, length of stay, and readmission after surgery for tibial plateau fractures. J Orthop Trauma 29(3):e121-e126

8. Canadian Orthopaedic Trauma S (2006) Open reduction and internal fixation compared with circular fixator application for bicondylar tibial plateau fractures. Results of a multicenter, prospective, randomized clinical trial. J Bone Jt Surg Am 88(12):2613-2623

9. Choo KJ, Morshed S (2014) Postoperative complications after repair of tibial plateau fractures. J Knee Surg 27(1):11-19

10. Cuellar-Avaroma A, King-Martinez AC, Hernandez-Salgado A, Torres-Gonzalez R (2006) Complications in complex fractures of the tibial plateau and associated factors. Cir Cir 74(5):351-357

11. Luo CF, Sun H, Zhang B, Zeng BF (2010) Three-column fixation for complex tibial plateau fractures. J Orthop Trauma 24(11):683-692

12. Prasad GT, Kumar TS, Kumar RK et al (2013) Functional outcome of Schatzker type V and VI tibial plateau fractures treated with dual plates. Indian J Orthop 47(2):188-194 
13. Qiu WJ, Zhan Y, Sun H et al (2015) A posterior reversed L-shaped approach for the tibial plateau fractures - a prospective study of complications (95 cases). Injury 46(8):1613-1618

14. Ruffolo MR, Gettys GF, Montijo HE et al (2015) Complications of high-energy bicondylar tibial plateau fractures treated with dual plating through 2 incisions. J Orthop Trauma 29(2):85-90

15. Haller JM, Kubiak EN (2013) Weight bearing after a periarticular fracture: what is the evidence? Orthop Clin N Am 44(4):509-519

16. Kubiak EN, Beebe MJ, North K et al (2013) Early weight bearing after lower extremity fractures in adults. J Am Acad Orthop Surg 21(12):727-738

17. Bailon-Plaza A, van der Meulen MC (2003) Beneficial effects of moderate, early loading and adverse effects of delayed or excessive loading on bone healing. J Biomech 36(8):1069-1077

18. Claes LE, Heigele CA, Neidlinger-Wilke C et al (1998) Effects of mechanical factors on the fracture healing process. Clin Orthop Relat Res (355 Suppl):S132-S147

19. Gardner MJ, van der Meulen MC, Demetrakopoulos D et al (2006) In vivo cyclic axial compression affects bone healing in the mouse tibia. J Orthop Res 24(8):1679-1686

20. Ware JE, Kosinski M, Keller SD (1995) How to score the SF-12 physical and mental health summaries: a user's manual. New England Medical Centre, Boston

21. McCormack HM, Horne DJ, Sheather S (1998) Clinical applications of visual analogue scales: a critical review. Psychol Med 18:1007-1019

22. Schatzker J, Mcbroom R, Bruce D (1979) The tibial plateau fracture: the Toronto experience 1968-1975. Clin Orthop Relat Res 138:94-104
23. Warren CG, Lehmann JF (1975) Training procedures and biofeedback methods to achieve controlled partial weight bearing: an assessment. Arch Phys Med Rehabil 56(10):449-455

24. Thomas G, Whalley H, Modi C (2009) Early mobilization of operatively fixed ankle fractures: a systematic review. Foot Ankle Int 30(7):666-674

25. Swart E, Bezhani H, Greisberg J et al (2015) How long should patients be kept non-weight bearing after ankle fracture fixation? A survey of OTA and AOFAS members. Injury 46:1127-1130

26. Black JD, Bhavikatti M, Al-Hadithy N et al (2013) Early weightbearing in operatively fixed ankle fractures: a systematic review. Foot 23(2-3):78-85

27. Solomon LB, Callary SA, Stevenson AW et al (2011) Weightbearing-induced displacement and migration over time of fracture fragments following split depression fractures of the lateral tibial plateau: a case series with radiostereometric analysis. J Bone Jt Surg Br 93(6):817-823

28. Westerman RW, Hull P, Hendry RG, Cooper J (2008) The physiological cost of restricted weight bearing. Injury 39(7):725-727

29. JJ dM (2009) Dynamics of human connective tissue. Bohn Stafleu van Loghum, Houten

30. Kalmet PHS, Meys G, Horn V YY et al (2018) Permissive weight bearing in trauma patients with fracture of the lower extremities: prospective multicenter comparative cohort study. BMC Surg 18(1):8 